\title{
Evaluación pre-post de una actividad formativa sobre alimentación y actividad física dirigida a estudiantes universitarios del campus de Melilla
}

\author{
Pre-post evaluation of a training activity on food and physical \\ activity addressed to university students of the Melilla campus
}

\author{
Elisabet Fernández Gómez ${ }^{1}$, María Angustias Sánchez-Ojeda ${ }^{1}$, Carmen Enrique Mirón ${ }^{2 *}$ y Adelina Martín-Salvador ${ }^{1}$ \\ ${ }^{1}$ Departamento de Enfermería. Facultad de Ciencias de la Salud. Campus de Melilla (España). \\ ${ }^{2}$ Departamento de Química Inorgánica. Facultad de Ciencias de la Educación y del Deporte. Campus de Melilla (España).
}

\begin{abstract}
Resumen: Las enfermedades crónicas no transmisibles son producidas por un estilo de vida no saludable, como una mala alimentación y no realizar actividad física. El conocimiento sobre alimentación y ejercicio físico de los estudiantes universitarios del ámbito sanitario y educativo es imprescindible para la promoción de la salud y la prevención de enfermedades. El objetivo del estudio ha sido conocer cómo influye en los estudiantes una actividad formativa extracurricular en el conocimiento sobre alimentación y actividad física. Mediante un diseńo pre-post se analizan las respuestas dadas a un cuestionario ad hoc. Los resultados evidencian el incremento del conocimiento tras la intervención educativa, siendo los alumnos de Enfermería los que alcanzan mejores puntuaciones. Las actividades formativas extracurriculares son un medio eficaz para adquirir y reforzar, de manera positiva y altamente motivacional, los conocimientos de los universitarios permitiendo así su labor en el ámbito de la educación para la salud Palabras clave: Estudiantes universitarios, conocimientos, nutrición, actividad física, actividad formativa
\end{abstract}

Abstract: Chronic non-communicable diseases are produced by an unhealthy lifestyle, like a poor diet and not perform physical activity. The knowledge about the diet and the physical exercise of the university students of the sanitary and educative field is essential for the promotion of the health and the prevention of illnesses. The objective of the study was to know how an extracurricular training activity influences the students in the knowledge about the nutrition and the physical activity. Through a pre-post design the answers given to an ad hoc questionnaire are analyzed. The results show the increase in knowledge after the educational intervention, with nursing students achieving the best scores. The extracurricular training activities are an effective instrument to acquire and reinforce, in a positive and highly motivational way, the knowledge of university students thus allowing their work in the field of health education.

Keywords: University Students, Knowledges, Nutrition, physical activity, formative activity

\section{Introducción}

El aumento del sobrepeso y de la obesidad es una preocupación mundial. Según la Organización Mundial de la Salud, en 2016, el 39\% de las personas adultas presentaban sobrepeso y el 13\% obesidad (OMS, 2016). En España, el 17,4\% de la población padece obesidad y el $37,1 \%$ sobrepeso, por lo que más del 50\% de la población mayor de 18 años tienen exceso de peso, además la prevalencia de sedentarismo es del 36\% (Ministerio de Sanidad, Consumo y Bienestar Social, 2017).

A esta situación, hay que unir las alteraciones que se están produciendo en los estilos de vida de nuestro entorno como el cambio de la dieta mediterránea por comidas rápidas, el aumento de las horas de trabajo, el estrés, la poca actividad física y en general hábitos de vida poco saludables, han provocado un incremento de la mayoría de las enfermedades crónicas no transmisibles, teniendo estas una gran relevancia en los países occidentales, produciendo una alta morbimortalidad y discapacidad, además de la repercusión socioeconómica que ellas conlleva (Banegas, Rodríguez-Artalejo y Graciani, 2002).

Dirección para correspondencia [Correspondence address]: Carmen Enrique Mirón, Dirección: C/Santander, 152057 Melilla (España). E-mail: cenrique@ugr.es
Las enfermedades crónicas no transmisibles son un grupo diverso de dolencias producidas por factores de riesgo evitables como la alimentación desequilibrada, obesidad, sedentarismo, así como el tabaquismo y consumo de alcohol. Entre ellas podemos citar la diabetes, enfermedades cerebrovasculares, cardiopatías, cáncer y enfermedades respiratorias crónicas y todas ellas se presentan como las principales causas de mortalidad en el mundo (Vargas, Barragán, Peralta, Hernández, Ontiveros et al., 2015).

El estilo de vida que presentan los jóvenes determina un conjunto de actitudes hacia determinados productos alimenticios, los cuales algunas veces pueden ser saludables y otras veces nocivos para la salud (Díaz, Carus, Macossay y Barbosa, 2010).

La Organización Mundial de la Salud, en su informe sobre la salud en el mundo, describe los diez principales factores de riesgo para la salud, los cuales son cruciales para el desarrollo de las enfermedades crónicas no transmisibles y cinco de estos factores de riesgo están estrechamente relacionados con la alimentación y el ejercicio físico: obesidad, sedentarismo, hipertensión arterial, hipercolesterolemia y consumo insuficiente de frutas y verduras (OMS, 2002). 
La promoción de hábitos saludables es la principal clave para evitar o reducir las enfermedades crónicas no transmisibles (Kotseva, 2008). Existen evidencias científicas en torno a la repercusión positiva que sobre la salud tienen el aumento de la práctica de ejercicio físico y la mejora de la alimentación (Smith, Bauman, Bull, Booth y Harris, 2000; Elley, Kerse, Arroll y Robinson, 2003).

Es necesario concienciar a la población de la importancia de llevar a cabo hábitos de vida saludables para prevenir enfermedades. Esta promoción de hábitos saludables, especialmente la actividad física y la alimentación, es una labor primordial de los profesionales de la salud y de la educación, por lo que es esencial que los futuros profesionales de la rama de ciencias de la salud y de ciencias de la educación, actividad física y deporte adquieran conocimientos en hábitos saludables, ya que en un futuro próximo tendrán que llevar a cabo educación sanitaria y serán los responsables de promover estilos de vida saludables a las generaciones futuras.

Actualmente, en la Universidad de Granada el Grado en Enfermería tiene entre sus materias de formación básica una asignatura de Nutrición y Dietética con 6 ECTS en el segundo curso. Mediante la impartición de esta asignatura los estudiantes de Enfermería adquieren como competencias específicas, entre otras, "Conocer y valorar las necesidades nutricionales de las personas sanas y con problemas de salud en el ciclo vital, para promover y reforzar pautas de conducta alimentaria saludable. Identificar los problemas nutricionales de mayor prevalencia y seleccionar las recomendaciones dietéticas adecuadas" (Verifica, 2008). En el Grado en Fisioterapia es una asignatura optativa, de 6 créditos ECTS, y se imparte en el segundo semestre del segundo curso, siendo una de las competencias a alcanzar "Promover hábitos de vida saludables a través de la educación para la salud” (Verifica, 2008). En el Grado en Ciencias de la Actividad Física y el Deporte también es optativa con 6 créditos ECTS y las competencias específicas a alcanzar son: "Conocer y valorar las necesidades nutricionales de las personas sanas y con problemas de salud a lo largo del ciclo vital, para promover y reforzar pautas de conducta alimentaria saludable. Identificar los problemas nutricionales de mayor prevalencia y seleccionar las recomendaciones dietéticas adecuadas" (Verifica, 2013).

Numerosos estudios han demostrado que poseer conocimientos de hábitos saludables no implica que los estudiantes universitarios lleven a la práctica un modelo de vida saludable, sobre todo en lo relativo a alimentación y actividad física, incluso en aquellos estudiantes que cursan estudios relacionadas con la salud (Badir, Tekkas y Topan, 2014; Gómez y Salazar, 2010; Gopalakrishnan, Ganeshkumar, Prakash, Christopher y Amalraj, 2014; Rizo, González y Cortés, 2014;), pero es decisivo que estos universitarios, que en un futuro tienen que aplicar medidas de promoción de la salud a la población en general, adopten comportamientos saludables en cuanto a alimentación y ejercicio físico, ya que deben ser ejemplos a seguir a través de sus propias conductas.

\section{Justificación}

Los estudios planteados sobre el conocimiento vinculado a alimentación y actividad física en los adolescentes universitarios son limitados; estando la mayoría de ellos relacionados con los estilos de vida saludable y hábitos alimentarios. Hay, por tanto, un déficit de investigaciones destinadas al análisis de la efectividad de actividades formativas extracurriculares a pesar del elevado número de ellas que se realizan en el ámbito universitario. En este trabajo se trata de aportar datos al respecto.

\section{Objetivo}

Evaluar la efectividad de una acción formativa sobre alimentación y actividad física dirigida a estudiantes universitarios de los Grados en Enfermería (GE), Fisioterapia (GF) y Educación Primaria-Ciencias de la Actividad Física y del Deporte (DG) del Campus de Melilla (Universidad de Granada).

\section{Material y método}

Se ha llevado a cabo un estudio de tipo descriptivo-correlacional y transversal pre-post, sin grupo control. A través de un cuestionario estructurado se realizó un pre-test, con el fin de analizar los conocimientos de los estudiantes universitarios sobre diferentes aspectos vinculados con alimentación, nutrición y actividad física antes de las intervenciones programadas en forma de charlas y talleres de 60-90 minutos de duración, para que después de estas intervenciones se volviese a pasar el mismo cuestionario post-test y comparar, de este modo, los resultados en los dos momentos.

\section{Muestra}

Para la selección de los sujetos se realizó un muestreo no probabilístico por conveniencia, asociado a los participantes en la intervención formativa. El criterio de selección utilizado fue ser alumno/a de los Grados asociados a la sanidad y a la educación y actividad física del Campus de Melilla (Universidad de Granada).

El total de participantes en la actividad formativa fueron 45 estudiantes de primer y segundo curso de los Grados de Enfermería (GE), Fisioterapia GF) y Educación PrimariaCiencias de la Actividad Física y del Deporte (DG) del Campus de Melilla (Universidad de Granada). La distribución por curso, género y titulación queda recogida en la tabla 1 .

Evaluación pre-post de una actividad formativa sobre alimentación y actividad... SPORT TK, 8(2) (supl. 1), 73-80 
Tabla 1. Distribución por curso, género y titulación de los participantes (pre-post).

\begin{tabular}{llc}
\hline & Variables & Frecuencia (Porcentaje) \\
\hline Género & Masculino & $24(53.3)$ \\
& Femenino & $21(46.7)$ \\
Curso & $1^{\circ}$ & $27(60.0)$ \\
& $2^{\circ}$ & $18(40.0)$ \\
Titulación & GE & $12(26.7)$ \\
& GF & $23(51.1)$ \\
& DG & $10(22.2)$ \\
\hline
\end{tabular}

Tal y como queda recogido en la tabla 1, el porcentaje de hombres $(53.3 \%)$ y de mujeres $(46.7 \%)$ es bastante similar. No ocurre igual respecto al curso siendo mayoritarios los alumnos de primero (60\%) frente a los de segundo (40\%). Finalmente, el mayor porcentaje de asistentes está realizando el GF (51.1\%), seguido de los alumnos del GE (26.7\%) y los que menos representación tienen son los alumnos que cursan el DG $(22.2 \%)$.

\section{Instrumento de medida}

El instrumento utilizado fue un cuestionario ad hoc de 13 ítems con respuesta múltiple y una sola opción verdadera, 8 dirigidos a evaluar conocimientos relacionados con alimentación en general (alimentación saludable, etiquetado nutricional, dietas y mitos nutricionales y valoración nutricional) y 5 enfocados a temas de alimentación y actividad física tales como alimentación-ejercicio, suplementación nutricional y ayuno (Anexo 1). Asimismo, incluía los datos de identificación reseñados anteriormente (ver tabla 2).

El pre-test se pasó inmediatamente antes de iniciar la actividad formativa y el post-test al finalizar la misma. En ambos casos, los participantes fueron los mismos por lo que los datos de identificación se mantuvieron.

Tabla 2. Bloques temáticos y número de ítems.

\begin{tabular}{lc}
\hline Bloques temáticos & Número (Ítems) \\
\hline Datos de identificación & 3 \\
Conocimientos alimentarios & 7 (P1-P6, P8-P9) \\
Conocimientos deportivos & 6 (P7, P10-P13) \\
\hline
\end{tabular}

\section{Variables de estudio}

En este estudio se han establecido como variables independientes las sociodemográficas o de identificación, en concreto: sexo, curso académico y titulación a la que pertenecen. Como tales variables independientes son de tipo atributivas puesto que no se pueden manipular. Las variables dependientes, son las respuestas dadas a los ítems del cuestionario.

\section{Descripción de la actividad}

La intervención educativa, compuesta por clases teóricas, talleres, charlas y foros, tuvo lugar en la semana del 12 al 16 de noviembre de 2018. Se comenzó con la recepción de los participantes seguida de la inauguración de la actividad formativa que dio paso a la primera conferencia en donde se abordaron aspectos básicos y fundamentales sobre alimentación, grupos de alimentos, así como las recomendaciones para establecer una dieta saludable. Seguidamente, se desarrolló un taller sobre etiquetado nutricional en donde los participantes aprendieron a interpretar correctamente la información recogida en las etiquetas que acompañan a los productos alimenticios.

Las intervenciones del día siguiente giraron en torno a los mitos y creencias sobre alimentación y nutrición así como sobre las dietas milagro y las consecuencias para la salud que puede acarrear su seguimiento.

El tercer día estuvo dedicado a la alimentación asociada al ejercicio físico. Dicho tema se abordó en una conferencia inicial que fue seguida de un taller sobre valoración nutricional en el cual los alumnos se familiarizaron con las medidas antropométricas y el diseño de dietas personalizadas en función del sexo, la edad, el nivel de actividad física, preferencias, etc. El abordaje de la actividad física continuó en la jornada siguiente con un taller sobre valoración funcional y la práctica de diferentes tipos de ejercicio así como con la ponencia destinada al tratamiento de la suplementación nutricional relacionada con el incremento del rendimiento deportivo y sus efectos.

La última jornada estuvo dedicada al ayuno, sus efectos, tipos, fisiología, beneficios y riesgos de la práctica del ayuno intermitente y sus consecuencias. Tras la conferencia se desarrolló un foro grupal en donde los participantes tuvieron la oportunidad de participar y aclarar todas aquellas dudas que les hubieran surgido a lo largo de las sesiones. Tras el foro, se procedió a la clausura de la actividad formativa.

\section{Análisis de los datos}

Para el análisis estadístico de los datos se utilizó el programa SPSS 24.0 para Windows. Las variables de identificación se expresaron en frecuencias absolutas y porcentajes al igual que las variables del cuestionario que fueron tratadas como variables cualitativas. Dado el tamaño muestral y el no ajuste a la normalidad (prueba de Kolmogorov-Smirnov) se utilizaron pruebas no paramétricas. Así, para la comparación de proporciones se utilizó la prueba de Chi-cuadrado y se consideró como valor de significación estadística una $\mathrm{p}<.05$.

Evaluación pre-post de una actividad formativa sobre alimentación y actividad... SPORT TK, 8(2) (supl. 1), 73-80 


\section{Resultados}

Los resultados obtenidos antes y después de la intervención educativa se recogen en la tabla 3 .

Del total de los ítems relacionados con alimentación (8), en 7 (88\%) se ha observado una mejora estadísticamente significativa después de la intervención y sólo en uno de los ítems se ha constatado un ligero empeoramiento. Llama la atención el escaso porcentaje de aciertos en la pregunta que alude al etiquetado nutricional (ítem 4). Aunque el $11 \%$ de aciertos en el pre-test se ve mejorado en el post-test (22\%), sigue siendo sig- nificativamente bajo. Situación similar se presenta en el ítem 6 vinculado a la distribución calórica de la ingesta que es respondida acertadamente por solo un $13 \%$ en el pre siendo, sin embargo, el ítem que mejores resultados relativos consigue en el pos-test. Asimismo, en el ítem 8, relacionado con el consumo de huevos, si bien acierta un tercio de los participantes en el pre-test, en el post-test algo más de la mitad (53.3\%) responde correctamente. Finalmente, es en el ítem 9 (relacionado con las dietas milagro) en donde se aprecia un ligero empeoramiento, pasando de un $88.9 \%$ de aciertos en el pre-test a un $86.7 \%$ en el post-test, sin llegar a ser significativo.

Tabla 3. Porcentaje de alumnos que responden correctamente a cada pregunta en el pre y post-test.

\begin{tabular}{|c|c|c|c|c|c|}
\hline \multirow[b]{2}{*}{ Items } & \multicolumn{2}{|c|}{$\%$ respuestas correctas } & \multirow{2}{*}{ Valor $\mathrm{p}$} & \multirow{2}{*}{$\%$ cambio de respuestas correctas } & \multirow{2}{*}{ Valoración de la efectividad } \\
\hline & Pre & Post & & & \\
\hline \multicolumn{6}{|c|}{ Conocimientos sobre alimentación en general } \\
\hline 1 & $44.4 \%$ & $95.6 \%$ & .000 & 48.8 & Mejora \\
\hline 2 & $68.9 \%$ & $91.1 \%$ & .001 & 24.4 & Mejora \\
\hline 3 & $60.0 \%$ & $91.1 \%$ & .003 & 31.1 & Mejora \\
\hline 4 & $11.1 \%$ & $22.2 \%$ & .005 & 15.5 & Mejora \\
\hline 5 & $84.4 \%$ & $93.3 \%$ & .060 & 8.9 & Mejora \\
\hline 6 & $13.3 \%$ & $88.9 \%$ & .000 & 75.6 & Mejora \\
\hline 8 & $33.3 \%$ & $53.3 \%$ & .001 & 15.1 & Mejora \\
\hline 9 & $88.9 \%$ & $86.7 \%$ & .120 & $(-) 2.2$ & Empeora \\
\hline \multicolumn{6}{|c|}{ Conocimientos sobre alimentación y actividad física } \\
\hline 7 & $26.7 \%$ & $35.6 \%$ & .060 & 11.1 & Mejora \\
\hline 10 & $28.9 \%$ & $62.2 \%$ & .003 & 31.1 & Mejora \\
\hline 11 & $44.4 \%$ & $88.9 \%$ & .002 & 42.2 & Mejora \\
\hline 12 & $46.7 \%$ & $93.3 \%$ & .002 & 51.1 & Mejora \\
\hline 13 & $37.8 \%$ & $31.1 \%$ & .053 & $(-) 4.4$ & Empeora \\
\hline
\end{tabular}

En la figura 1 se presentan el total de aciertos por cada uno de los ítems correspondientes al bloque de "alimentación" en el pre y post-test.

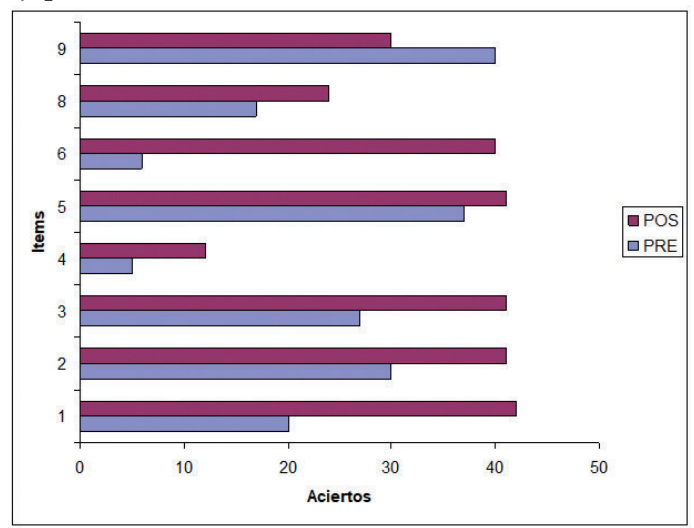

Figura 1. Total de aciertos pre y post-test en el bloque "Alimentación”.
En cuanto a los conocimientos sobre alimentación y actividad física, de 5 ítems mejoraron significativamente un $80 \%$ y $20 \%$ empeoró (tabla 1). Llama la atención el incremento de respuestas acertadas en el post-test en los ítems 10 (ejercicio físico de forma segura), 11 (suplementación en el ejercicio) y 12 (suplementación y rendimiento deportivo) superando en todos los casos un 30\% ( $\mathrm{p}=.003)$. Por el contrario, en el ítem 13 (ayuno y actividad física), el porcentaje de respuestas acertadas fue inferior en un $7 \%$ aproximadamente en el post-test frente al pre-test.

El total de aciertos correspondiente al bloque "Alimentación y actividad física" en el pre y post-test que recogido en la figura 2 . 


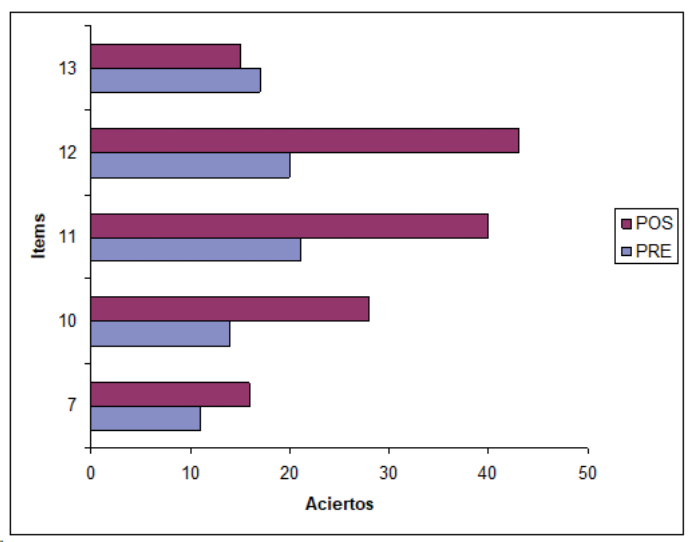

Figura 2. Total de aciertos pre y post-test en el bloque "Alimentación y actividad física”.

Respecto al género, no se ha encontrado asociación con el total de aciertos pre-test $\left(\mathrm{X}^{2}=.094, \mathrm{p}=.539\right)$, ni con el post-test $\left(\mathrm{X}^{2}=.101, \mathrm{p}=.509\right)$. Por el contrario, al contrastar los resultados con la variable titulación se pasa de no tener asociación entre el total de aciertos en el pre $\left(\mathrm{X}^{2}=-.186, \mathrm{p}=.222\right)$ a presentar una asociación estadísticamente significativa en el posttest $\left(\mathrm{X}^{2}=-.395, \mathrm{p}=.007\right)$, siendo los alumnos del GE los que consiguen mayores puntuaciones frente a los del GF y DG.

En cuanto al curso, se aprecia una correlación estadísticamente significativa y negativa en el pre-test $\left(\mathrm{X}^{2}=-.393\right.$, $\mathrm{p}=.008)$ siendo los estudiantes de primer curso los que consiguen un mayor número de aciertos, sin embargo no la hay en el post-test $\left(\mathrm{X}^{2}=.025, \mathrm{p}=.868\right)$ en donde no se aprecia asociación entre el total de aciertos y el curso del alumnado. Estos resultados ponen de manifiesto que la participación en dicha actividad formativa, ha permitido que los alumnos alcancen un nivel similar de conocimientos.

\section{Discusión}

Actualmente existen numerosas enfermedades asociadas a unos hábitos de vida inapropiados, por eso es importante la educación para la salud que permita aumentar la calidad de vida. En este sentido, con el Espacio Europeo de Educación Superior se iniciaron nuevos perfiles profesionales y titulaciones que permitieran una formación adecuada y competente para responder adecuadamente a estas exigencias. Así, en la actualidad, se dispone de titulaciones relacionadas con la salud, la educación y la actividad física y el deporte en donde se contemplan competencias que, hasta entonces, estaban restringidas a médicos especialistas siendo éstos los encargados de educar en aspectos nutricionales y deportivos a la sociedad.

Solís (2004) establece la comparativa de dos titulaciones que sostienen su parcela dentro de la nutrición deportiva, Diplomado en Ciencias de los Alimentos, Dietética y Nu- trición y Licenciado en Ciencias de la Actividad Física y el Deporte. La conclusión alcanzada es que ambas titulaciones requieren una formación específica sobre nutrición deportiva. En el presente estudio se recogen datos similares, y es que con la formación durante el periodo universitario, no sería suficiente para adquirir los conocimientos adecuados. Se ha comprobado la utilidad de actividades extracurriculares para la adquisición de conocimientos en materia de nutrición y deporte.

Aunque existe gran cantidad de información relativa a la alimentación y a la nutrición en los medios de comunicación y en la publicidad, los estudios que se basan en averiguar el nivel de conocimientos previos y su vínculo con estado nutricional son limitados (Pozo, Cubero y Ruiz, 2013).

El empleo de técnicas de enseñanza ajustadas al aprendizaje de los participantes ha sido usado en otras actividades formativas dirigidas a adolescentes en las que también se logró aumentar el nivel de conocimientos de los participantes (Sánchez Socarras, Fornons Fontdevila, Aguilar Martinez, Vaque Crusellas, y Mila Villarroel, 2015). Así, los resultados obtenidos en el estudio aquí presentado ponen de manifiesto que los alumnos han adquirido conocimientos sobre alimentación y actividad física después de la intervención formativa compuesta por diferentes técnicas de enseñanza-aprendizaje, al igual que dictaminan estos autores.

Existen numerosos estudios que demuestran que los estudiantes universitarios afirman tener conocimientos sobre alimentación saludable y la realización de actividad física, sin llegar a practicarlos (Arrivillaga, Salazar y Correa, 2003; Bernárdez, Castro, Collins y De La Montaña, 2013; Cervera et al., 2013; Montero, Úbeda y García, 2006; Romero, Cayuela, Molina, y Sousona, 2004), pero no hay muchas investigaciones que analicen los conocimientos previos y posteriores tras una intervención educativa, tal y como se presentas en este estudio, que aunque mejoró los conocimientos del alumnado se ha demostrado que son necesarias más intervenciones sobre todo en los GF y GA, ya que el GE tuvo más conocimiento, tal y como lo hicieron los autores Sánchez, Aguilar, González, Esquius y Vaqué (2017) en un grupo de estudiantes de Fisioterapia y Logopedia, en el que la asignatura de Nutrición no es una materia obligatoria.

Rodrigo Vega (2014) señala que la asignatura de nutrición en los Grados en Enfermería y en Educación mejora los conocimientos y la adherencia a la dieta mediterránea (ADM). A nivel de conocimientos sobre la dieta mediterránea se evidenciaron diferencias significativas en cuanto a las mejoras de las contestaciones en ambos grupos, sobretodo en el grupo de enfermería a pesar de partir con peores resultados. Además, se demostró que los cambios conceptuales suelen ser más notables que los cambios en los hábitos, por lo que deberían mejorar su ADM ambos grupos.

Los resultados de nuestro trabajo demuestran que acciones 
formativas sobre educación nutricional en forma de talleres y charlas, junto a materias curriculares contempladas en los respectivos planes de estudio, pueden ser un instrumento útil para aumentar conocimientos, e incluso, mejorar actitudes sobre estilos de vida saludables.

Entre las limitaciones a destacar en el presente trabajo se encuentra la del diseño prepost, ya que al no tener un grupo control resulta difícil determinar si los resultados realmente son debidos a la propia intervención o a la adquisición de información por otras fuentes durante la realización de la actividad.

Por último, haciendo referencia a la metodología de talleres y charlas, según Portero, Cirne, Mathieu (2002), las acciones informativas/ formativas deben contemplar tres aspectos fundamentales: 1 . Coherencia (correlación entre temática e institución). 2. Constancia (mayor frecuencia de actividades y periodicidad predeterminada de las mismas). 3. Extensión (cobertura masiva e inclusión del mayor número de centros). De estos tres aspectos, el único que no cumple nuestra actividad es el segundo, ya que se ha tratado de una intervención puntual dentro del curso académico. Ahora bien, dados los buenos resultados, sin duda volverá a repetirse. En cuanto al tercer aspecto, ha sido una actividad ofertada a todo el alumnado del Campus y por ende a todos los centros que en él imparten docencia.

\section{Conclusiones}

Los resultados obtenidos permiten concluir que las intervenciones formativas en ámbitos no formales sobre nutrición y actividad física mejoraron los conocimientos del alumnado participante. El ambiente mas distendido, la falta de presión generada por el entorno del aula, la participación de profesionales en la materia, entre otros aspectos, mejoran la motivación y por tanto la receptividad. Así, tras la participación, todos los alumnos, independientemente del curso académico que estén cursando, se igualan en conocimientos. No obstante, se pone de relieve la influencia de la titulación cursada que puede propiciar una mayor atención derivada del área competencial de la misma como es el caso de los estudiantes del Grado de Enfermería que alcanzan mejores resultados.

\section{Referencias}

1. Arrivillaga, M., Salazar, I.C., Correa, D. (2003). Creencias sobre la salud y la relación con las prácticas de riesgo o de protección en jóvenes universitarios. Colombia Médica, 34, 186-195.

2. Badir, A., Tekkas, K., y Topán, S. (2015). Knowladge of cardiovascular disease in Turkish undergraduate nursing students. European Journal of Cardiovascular Nursing, 14(5), 441-449. doi: https://doi. org/10.1177/1474515114540554

3. Banegas, J. R., Rodríguez-Artalejo, F., y Graciani, A. (2002). Interacción de los factores de riesgo en las enfermedades crónicas. Revista Española de Salud Pública, 76, 01-05.

4. Bernárdez, M., Castro, L., Collins, A., y De La Montaña, J. (2013). Variaciones en la dieta de universitarios gallegos (campus de Ourense) con relación al patrón cardioprotector de la dieta mediterránea. Nutrición Hospitalaria, 28(6), 2099-2016.

5. Cervera, F., Serrano, R., Vico, C., Milla, M., y García, M.J. (2013). Hábitos alimentarios y evaluación nutricional en una población universitaria. Nutrición Hospitalaria, 28(2), 438-446.

6. Díaz, R., Carús, M., Macossay, C., y Barbosa, I. (2010). Efecto del estilo de vida en los hábitos y conductas alimentarias en universitarios. Revista Exploratoris, 1, 1-6.

7. Elley, C.R., Kerse, N., Arroll, B., y Robinson, E. (2003). Effectiveness of counselling patients on physical activity in general practice: cluster randomised controlled trial. British Medical Journal, 326(7393), 793. doi: 10.1136/bmj.326.7393.793

8. García Solis, M.A. (2004). El diplomado en nutrición y el licenciado en ciencias de la actividad física y el deporte en el ámbito de la nutrición deportiva. Revista Internacional de Medicina y Ciencias de la Actividad Física y el Deporte, 4 (13), 58-72.

9. Gómez, J.I., y Salazar, N. (2010). Hábitos alimenticios en estudiantes universitarios de ciencias de la salud de Minatitlán. Tesis inédita de Doctorado. Universidad Veracruzana. Facultad de Enfermería, Minatitlán, México.

10. Gopalakrishnan, S., Ganeshkumar, P., Prakash, M. V., y Amalraj, V. (2012). Prevalence of overweight/obesity among the medical students, Malaysia. The Medical Journal of Malaysia, 67(4), 442-444.
11. Kotseva, K. (2008). Políticas preventivas globales. Estrategias a escala europea y mundial. Revista española de cardiología, 61(9), 960-970.

12. Ministerio de Sanidad, Consumo y Bienestar Social. (2017). Encuesta Nacional de Salud de España 2017. Recuperado de: https://www.mscbs. gob.es/estadEstudios/estadisticas/encuestaNacional/encuesta2017.htm.

13. Organización Mundial de la Salud (2002). Informe de Salud Mundial: 2002: Factores de Riesgo, Promoción de Vida Sana. Ginebra, Organización Mundial de la Salud.

14. Organización Mundial de la Salud (16 de febrero de 2018). Obesidad $y$ sobrepeso. Recuperado de: https://www.who.int/es/news-room/factsheets/detail/obesity-and-overweight.

15. Portero López P, Cirne Lima R, Mathieu G. (2002). La intervención en adolescentes y jóvenes en la prevención y promoción de la salud. Revista Española de Salud Pública, 76,577-584.

16. Pozo, A., Cubero, J., y Ruiz, C. (2013). Previous knowledge in food and nutrition and its relationship to the body mass index of a group of students in a penitentiary centre Spanish; a resource in health education. ENSAYOS. Revista de la Facultad de Educación de Albacete 28, 87-100.

17. Rizo, M.M., González, N.G, y Cortés, E. (2014). Calidad de la dieta y estilos de vida en estudiantes de ciencias de la salud. Nutrición Hospitalaria, 29(1), 153-157.

18. Rodrigo Vega, M., Ejeda Manzanera, J. M., González Panero, M. D. P., Gurruchaga, M., y Teresa, M. (2014). Cambios en la adherencia a la dieta mediterránea en estudiantes de los Grados de Enfermería y de Magisterio tras cursar una asignatura de Nutrición. Nutrición Hospitalaria, 30(5), 1173-1180.

19. Romero, A., Cayuela, Ml, Molina, A., y Sousona, M. (2004). ¿Se alimentan correctamente nuestros jóvenes universitarios? Rol de Enfermería, 27(2), 134.

20. Sanchez Socarras, V., Fornons Fontdevila, D., Aguilar Martinez, A., Vaque Crusellas, C., y Mila Villarroel, R. (2015). Evolution of knowledge about eating disorders through an educational intervention in post-compulsory students. Nutrición clínica y dietética hospitalaria, 35(1), 32-41. 
21. Sánchez, V., Aguilar, A., González, F., Esquius, L., y Vaqué, C. (2017). Evolución en los conocimientos sobre alimentación: una intervención educativa en estudiantes universitarios. Revista chilena de nutrición, 44(1), 19-27. doi: 10.4067/S0717-75182017000100003

22. Smith, B.J., Bauman, A.E., Bull, F.C., Booth, M.L., y Harris, M.F. (2000). Promoting physical activity in general practice: a controlled trial of written advice and information materials. British Journal of Sports Medicine, 34, 262-267.

23. Vargas, M. R., Barragán Hernández, O., Peralta Peña, S. L., Hernández Villa, E. A., Ontiveros Pérez, M., Favela Ocaño, M. A.,y Acuña Ruiz, M. J. Y. (2016). Factores de riesgo de enfermedades no transmisibles en académicos universitarios. Biotecnia, 18(1), 3-8.
24. Verifica. (2008). Agencia Nacional de Evaluación de la Calidad y Acreditación. Grado en Enfermería de la Universidad de Granada. Recuperado de http://grados.ugr.es/enfermeria/pages/infoacademica/verificaenfermeria/!

25. Verifica. (2008). Agencia Nacional de Evaluación de la Calidad y Acreditación. Grado en Fisioterapia de la Universidad de Granada. Recuperado de https:/grados.ugr.es/fisioterapia/pages/infoacademica/ verificafisioterapia/!

26. Verifica. (2013). Agencia Nacional de Evaluación de la Calidad y Acreditación. Grado en Ciencias de la Actividad Física y del Deporte por la Universidad de Granada. Recuperado de https:/oficinavirtual.ugr.es/ ConectaDocumenta/verDocumento.jsp

\section{Anexo 1}

\section{Cuestionario sobre alimentación y actividad física}

\section{CAMPUS DE MELILLA. UGR}

\section{Datos de identificación}

Sexo:

Curso:

Titulación:

\section{Por favor, conteste a las siguientes preguntas:}

1. En cuanto a la fruta, señale la correcta:

a) Después de las comidas y por la noche engorda.

b) No importa la hora que os comáis la pieza de fruta, lo que importa son las calorías totales ingeridas.

c) Son ricas en agua y el resto suele ser azúcar en forma de glucosa.

d) No lo sé.

2. Señale la afirmación correcta:

a) Es más saludable el consumo de margarina al ser vegetal que el de mantequilla al ser animal.

b) Tiene más propiedades nutritivas el pan integral que el refinado.

c) Es más recomendable el consumo de queso curado que fresco.

d) No lo sé.

3. ¿Sabrías identificar el potenciador de sabor más tóxico para nuestro organismo?

a) E-120 carmines.

b) E-621 glutamato monosódico.

c) E-250 nitritos.

d) No lo sé.

4. Sobre el etiquetado nutricional, señale la respuesta incorrecta.

a) El orden de los ingredientes aparecen según su cantidad en el alimento.

b) Existe regulación legislativa sobre los productos 0.0 . c) Las industrias dan diferentes nombres a un mismo ingrediente para evitar que aparezcan en primer lugar los ingredientes más perjudiciales (azúcar, aceites vegetales...).

d) No lo sé.

5. ¿Qué hay que tener en cuenta a la hora de realizar una dieta?

a) Evaluación antropométrica y bioquímica.

b) Evaluación clínica y dietética.

c) Las dos anteriores son correctas

d) No lo sé.

6. ¿En qué toma del día el porcentaje de energía debe ser mayor?

a) Desayuno.

b) Comida.

c) Indiferente, según las necesidades de cada organismo.

d) No lo sé.

\section{Señale la afirmación falsa:}

a) El deportista debe consumir una dieta lo más variada posible.

b) El deportista durante el ejercicio no debe hidratarse más allá de lo que le indique su mecanismo de la sed.

c) Los deportistas no deben tomar mayores cantidades de sal en la dieta pese a la mayor sudoración que presentan.

d) No lo sé.

8. En cuanto al consumo de huevos, señale la falsa:

a) La ovoalbúmina es la principal proteína de la clara, es la proteína patrón o de referencia porque está 100\% compuesta por todos los aa esenciales.

b) Es una fuente importante de todos los nutrientes (excepto HC, fibra, Vitamina C y carotenos).

c) El consumo de huevo eleva los niveles de colesterol en sangre, por lo que consumir más de 2 huevos a la semana resulta perjudicial.

d) No lo sé. 
9. En cuanto a las dietas milagros, señale la correcta:

a) Para realizar una dieta debes adaptarte a ella.

b) Hay veces que en las buenas dietas para adelgazar se necesita introducir algunos suplementos.

c) Una dieta en sí no determina prevenir una enfermedad, adelgazar o el tratamiento de una enfermedad, son procesos que dependen de muchos factores, atribuir el resultado final a sólo uno de ellos es inadecuado.

d) No lo sé.

10. En la prescripción de ejercicio físico de forma segura, es cierto que:

a) El paciente diabético debe realizar ejercicios de fuerza de alta carga para mejorar su control diabetológico.

b) La hipertensión es una contraindicación para la práctica de cualquier disciplina deportiva.

c) La disciplina HIIT puede no ser la mejor opción para la pérdida de peso debido a la poca adherencia que presenta.

d) No lo sé.

11. Señale la afirmación verdadera con respecto a la suplementación en el ejercicio:

a) Las personas que practican cualquier disciplina deportiva deberían tomar multivitamínicos para prevenir enfermedades metabólicas. b) La cafeína puede considerarse una ayuda ergogénica no dopante para los deportistas.

c) La L-carnitina potencia la quema de grasas en personas que practican ejercicio.

d) No lo sé.

12. Señale los suplementos nutricionales que han demostrado aumentar el rendimiento deportivo:

a) Creatina en deportes de explosión.

b) La taurina retrasa el inicio de la fatiga física.

c) Enzima Q10 oral en carrera.

d) No lo sé.

13. El ayuno (señala la falsa):

a) Aumenta el rendimiento tanto físico como mental debido a la creación de cuerpos cetónicos, que son el mejor combustible para el músculo y el cerebro.

b) Los últimos estudios ponen de manifiesto que ayunar durante algunos periodos cortos de tiempo pueden ayudar a perder peso y a mejorar el perfil cardiovascular (glucemia, lípidos en sangre...).

c) Uno de los riesgos del ayuno es la pérdida de masa magra.

d) No lo sé. 